\title{
Software Package STATISTICA and Educational Process
}

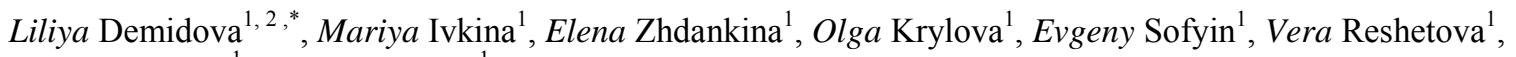 \\ Nikita Stepanov ${ }^{1}$ and Nikita Tyart ${ }^{1}$ \\ ${ }^{1}$ Ryazan State Radio Engineering University, 390005, Ryazan, 390005Russia \\ ${ }^{2}$ Moscow Technological Institute, 199334, Moscow, Russia
}

\begin{abstract}
The paper describes the main aspects of application of the software package STATISTICA in the educational process. Technologies of data mining which can be useful for students researches have been considered. The main tools of these technologies have been discussed.
\end{abstract}

\section{Introduction}

STATISTICA is an advanced analytics software package originally developed by StatSoft, which was acquired by Dell in March, 2014 [1, 2]. STATISTICA originally derives from a set of software packages and add-ons that were initially developed during the Mid1980 's by StatSoft. Nowdaws we can work with STATISTICA 13.0 (Release date: September 2015).

STATISTICA provides data analysis, data management, statistics, data mining, machine learning, text analytics and data visualization procedures.

The software includes a variety of predictive modeling, clustering, classification, and exploratory techniques. Additional techniques are available through integration with the free, open source R programming environment [3].

Operation of the software typically involves loading a table of data and applying statistical functions from pull-down menus or from the ribbon bar. The menus then prompt for the variables to be included and the type of analysis required. It is not necessary to type command prompts. Each analysis may include graphical or tabular output and is stored in a separate workbook [3].

Currently different educational courses will require students to have access to modern statistical software packages.

Software Package STATISTICA fully satisfies to requirements of educational process. In particular, this package allows to get acquainted easily with the principles of data mining and machine learning. Then students can use the acquired skills for their scientific researches. A herewith, there is no need for writing of difficult program codes.

\section{STATISTICA and Data Mining}

Data mining is an analytic process designed to explore large amounts of data in search of consistent patterns and/or systematic relationships between variables, and then to validate the findings by applying the detected patterns to new subsets of data [4].

This analytic process consists of the following main steps: initial exploration; model building or pattern identification with validation/verification; deployment (i.e., the application of the model to new data in order to generate predictions) [4]. But at first it is necessary to carry out the precise formulation of the problem.

The step of initial exploration usually starts with data preparation that may involve cleaning data, data transformations, selecting subsets of records. Also, in the case of data sets with large numbers of variables, this step suggests performing some feature selection operations to bring the number of variables to a manageable range. Besides, at this step we can use a wide variety of graphical and statistical methods in order to identify the most relevant variables and determine the complexity and/or the general nature of models that can be taken into account at the next step.

The step of model building or pattern identification involves considering various models and choosing the best one based on their predictive performance. At this step we can use a variety of techniques, many of which are based on so-called «competitive evaluation of models», that is, applying different models to the same data set and then comparing their performance to choose the best. These techniques, which are often considered the core of Predictive Data Mining, include: Bagging (Voting, Averaging), Boosting, Stacking (Stacked Generalizations), and Meta-Learning [4].

The final step of deployment involves using the model selected as best in the previous step and applying it to new data in order to generate predictions or estimates of the expected outcome [4].

Data mining is based on the conceptual principles of statistics including the traditional Exploratory Data Analysis and modeling. However, nowdays we can speak about the huge interest in developing new analytic techniques such as Classification Trees, General Classification and Regression Trees, General CHAID

Corresponding author: demidova.liliya@gmail.com 
Models, General CHAID Models, MARSplines, Neural Networks, Generalized EM and K-Means Cluster Analysis, Association Rules, Machine Learning (Bayesian, Support Vectors and Nearest neighbors), Random Forests for Regression and Classification, Generalized Additive Models, Feature Selection and Variable Screening and so on $[1,5]$.

We can use these analytic techniques to solve the different problems. A herewith the time expenditures for the required decision's obtaining are minimum that is convenient for the users who aren't owning good skills of programming. However, in STATISTICA there is no possibility of the «thin» control of parameters for many algorithms. Therefore STATISTICA can be considered as the tools of the preliminary analysis of some problem with application of the chosen technologies of data mining. Then, the own program realization of the corresponding algorithms or the use of the ready software packages is expedient.

Supervised learning is the machine learning problem of deducing a function from labeled training data. The training data set consists of training examples. A herewith each example is a pair containing an input object (usually a vector) and a desired output value. The supervised learning algorithm analyzes the training data set, evaluates a potential solution and produces an deduced function, which can be used for classifying the new objects. Classification and Regression are the most known techniques of supervised learning.

Unsupervised learning is the machine learning problem of deducing a function to describe hidden structure from unlabeled data. The supervised learning algorithm can't evaluate a potential solution, since the training examples are unlabeled. Unsupervised learning is closely related to the problem of density estimation in statistics. Cluster analysis and Principal component analysis are the most known techniques techniques of unsupervised learning.

To develop the classification and regression models we must carry out the following steps: to built the model describing a predetermined set of data classes; to estimate the predictive accuracy of the model; to use the model to classify new data for which the class label is unknown, if the predictive accuracy of the model is acceptable.

We can use different kinds of Classification and Regression techniques in STATISTICA, including: Classification and Regression, through STATISTICA Automated Neural Network; General Classification and Regression tree; General CHAID model; Boosted Tree Classification and Regression; Random Forest for Classification and Regression, etc.

Usually, the interpretation of results summarized in a tree is very simple. This simplicity is useful for the rapid classification of the new examples (objects). Besides, when analyzing any problems (for example, business problems), it is much easier to present a few simple logical «if-then» statements to management, than some elaborate complex equations.

The General Classification and Regression Trees (CART) module in STATISTICA allows building the classification and regression trees for categorical predictor variables (classification) and predicting continuous dependent variables (regression). This module supports the classic CART algorithm and includes various methods for pruning and crossvalidation, as well as the powerful v-fold crossvalidation methods.

We can use the example data file IrisSNN.sta, which is located in the /Example/Datasets directory of STATISTICA, to build the Classification Tree. This file contains the lengths and widths of sepals and petals of three types of irises (Setosa, Versicol, Virginic). The purpose of the classification analysis is to understand how one can discriminate between the three types of flowers, based on the four measures of width and length of petals and sepals. The classification tree will determine a set of logical «if-then» statements for classifying examples (objects). We can use the example data file Poverty.sta, which is located in the /Example/Datasets directory of STATISTICA , to build the Regression Tree. This file contains contains 1960 and 1970 Census figures for a random selection of 30 counties. The purpose of the classification analysis is to determine the correlates of poverty. As a result, we can find the variables that best predict the percent of families below the poverty line in a county.

Machine Learning tools in STATISTICA provides a number of advanced statistical methods (such as KNearest Neighbors (KNN), Naive Bayes Classifiers, Support Vector Machines (SVM)) for handling regression and classification problems with multiple dependent and independent variables (Figure 1).

STATISTICA K-Nearest Neighbors is a memorybased model defined by a set of examples (objects) for which the outcome are known (i.e., the examples are labeled) [6]. In K-Nearest Neighbors the independent and dependent variables can be either continuous or categorical. The problem is the regression for continuous dependent variables, otherwise the problem is the classification.

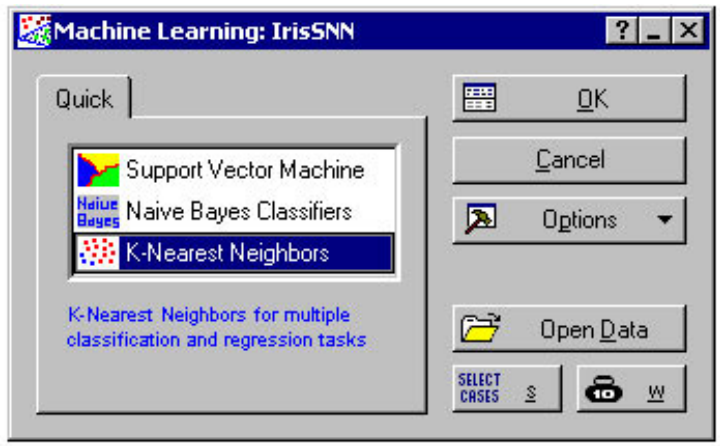

Fig. 1. Machine Learning Startup Panel

Hence, K-Nearest Neighbors in STATISTICA can handle both regression and classification problems.

If we have a new example (object), we would like to estimate the outcome based on the KNN examples. To make the decision $\mathrm{KNN}$ must find $\mathrm{K}$ examples that are closest in distance to our new example (object). KNN predictions are based on averaging the outcomes of the 
$\mathrm{K}$ nearest neighbors for the regression problems. For the classification problems KNN uses the vote majority rule.

Value of $\mathrm{K}$ strongly influences the prediction accuracy. To find the optimal value for $\mathrm{K}$ we can use the cross-validation algorithm in STATISTICA.

STATISTICA Naïve Bayesian Classifiers are the statistical classifiers, based on applying Bayes' theorem with strong (naive) independence assumptions between the features. The naive assumption of class conditional independence allows reducing the computational cost. These classifiers can predict the examples' (objects') class membership probabilities [1,5].

STATISTICA Support Vector Machine solves the regression and classification problems by constructing nonlinear decision boundaries. SVM can show a large degree of flexibility in handling classification and regression problems of varied complexities. It can handle multiple continuous and categorical variables $[1,5,7]$.

SVM classifier uses the special function called the kernel, with which the data set has been converted from the original space of features into the higher dimension space with the construction of a hyperplane that separates classes. A herewith two parallel hyperplanes must be constructed on both sides of the separating hyperplane. These hyperplanes define borders of classes and have been situated at the maximal possible distance from each other. Vectors of the objects' features which are the nearest to the parallel hyperplanes are called support vectors. The SVM classifier supposes an execution of training, testing, and classification. Satisfactory quality of training and testing allows using the resulting SVM classifier in the classification of the new objects. The SVM classifiers have been applied for credit risk analysis, medical diagnostics, handwritten character recognition, text categorization, face detection, Earth remote sensing, etc. [8-10].

STATISTICA SVM supports 4 types of models (Classification SVM Type 1 (C-SVM classification); Classification SVM Type 2 (nu-SVM classification); Regression SVM Type 1 (epsilon-SVM regression); Regression SVM Type 2 (nu-SVM regression)) with the linear kernel function, polynomial kernel function, radial basis kernel function and sigmoid kernel function.

We can use the example data file IrisSNN.sta to develop the SVM classifier (Figure 2) and the example data file Poverty.sta to solve the regression problem on the base of SVM.

Using the Support Vector Machines dialog we can define our settings on the Quick tab, Sampling Tab and so on (Figure 2). Then we can train the SVM classifier.

When training is finished, the Support Vector Machine Results dialog is displayed (Figure 3). Here we can review the results of SVM training as well as predictions in the form of spreadsheets, reports, and graphs. In the Summary box we can see specifications of the SVM model (in particular, the number of support vectors and their types, and the kernel functions and their parameters). Also, we can see here the dependent and independent variable list, the values of the training constants (capacity, epsilon and nu), the cross-validation results (when applicable) as well as classification statistics for training, testing, and overall samples (when applicable).

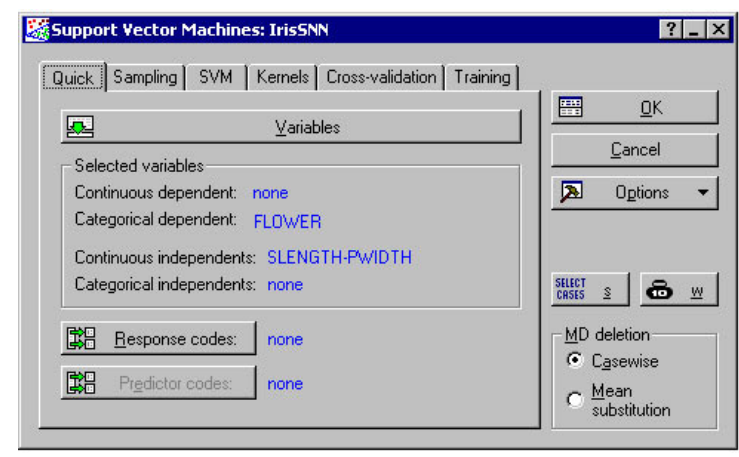

Fig. 2. Support Vector Machines dialog

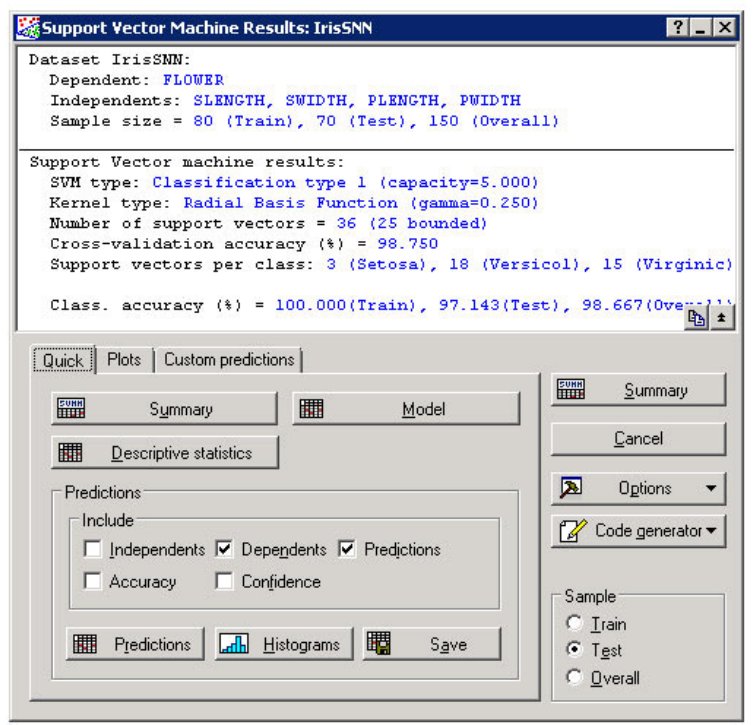

Fig. 3. Support Vector Machines Results dialog

We must evaluate the cross-validation estimates of the training constants (capacity, epsilon and nu). If any of these values are equal to their maximum crossvalidation range, this could be an indication that the search range was not large enough to include the best values. In this case, it is necessary to click the Cancel button to return to the Support Vector Machines dialog to enhance the search range. Also, it is useful to click the Model button to create spreadsheets which will contain information on the support vectors and coefficients. To obtain the additional information we can click the Descriptive statistics button. As a result, two spreadsheets containing the classification summary and confusion matrix will be created. Also, we can display the spreadsheet (and the corresponding histogram plots) of predictions via the Custom predictions tab (and include any other variable that might be of interest, e.g., independent, accuracy, etc., by selecting the respective option button on the Quick tab). The graphical review of the results can be made from the Plots tab where we can create two- and three-dimensional plots of the variables and confidence levels (Figure 4). 


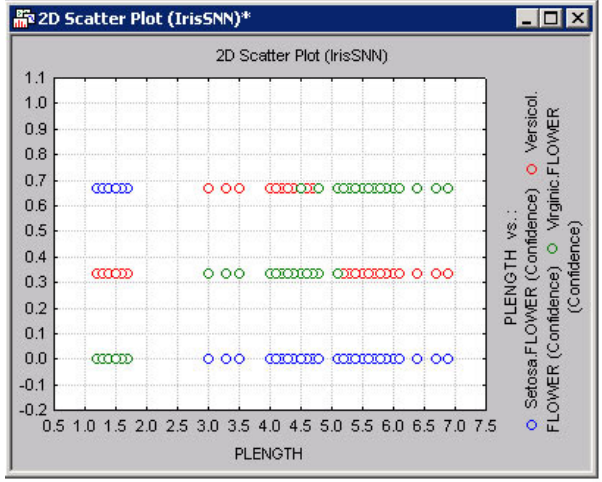

Fig. 4. Graphical review of the results

STATISTICA Support Vector Machine allows providing the full-fledged process of the SVM classifier development. A herewith the user has the access to the review of all variables and parameters of the process of the SVM classifier development. Therefore, STATISTICA Support Vector Machine is sufficient for development of the full-fledged SVM classifiers. Only in need of application into the development process of the additional user installations which aren't provided in STATISTICA Support Vector Machine, it makes sense to use more advanced software of the SVM classifier development. For example, it may be necessary, if we want to realize the simultaneous search of the kernel function type, values of the kernel function parameters and value of the regularization parameter for the SVM classifier on the base of evolutionary algorithms (for example, on the base of the modified PSO algorithm) $[11,12]$.

Cluster analysis is the process of grouping the data into classes or clusters so that objects within a cluster have high similarity in comparison to one another, but are very dissimilar to objects in other clusters. Clustering is one of the most utilised unsupervised learning techniques, where the learning don't rely on the predefined classes of the labeled training examples.

$\mathrm{K}-\mathrm{Means}$ algorithm is the basic clustering algorithm. This algorithm aims to partition $\mathrm{N}$ examples (objects) into $\mathrm{K}$ clusters in which each example (object) belongs to the cluster with the nearest mean, serving as a prototype of the cluster [13]. The basic version of KMeans algorithm is realized in the Cluster Analysis module.

The algorithms in the Generalized EM (expectation maximization) and K-Means Cluster Analysis module of STATISTICA extend the basic approach to clustering. These algorithms can be applied to both continuous and categorical variables. EM algorithm is an iterative algorithm for finding maximum likelihood or maximum a posteriori estimates of parameters [14]. It performs an expectation (E) step, which creates a function for the expectation of the log-likelihood evaluated, and a maximization (M) step, which finds parameters maximizing the expected log-likelihood found on the $\mathrm{E}$ step. These parameter-estimates are used to determine the distribution of the latent variables in the next E step.
The main shortcoming of K-Means clustering algorithm is that it is necessary to specify the number of clusters before starting the analysis (hence, the number of clusters must be known a priori). The Generalized EM and K-Means Cluster Analysis module uses a modified v-fold cross-validation scheme to determine the best number of clusters from the data.

These algorithms can be useful, for example, for the market research, biology researches and so on.

\section{Conclusions}

This paper has been discussed the main aspects of application of the data mining tools of the software package STATISTICA in the educational process. Users can easy use this package for scientific researches, as the extensive knowledges in the programming and the applied mathematics aren't required.

\section{References}

1. Statistica-Release Notes and Guides. support.software.dell.com. Retrieved 2015-09-23.

2. http://software.dell.com/products/statistica/

3. https://en.wikipedia.org/wiki/Statistica

4. http://documentation.statsoft.com/STATISTICAHel p.aspx? ? path=common/DataMining/DataMining

5. http://www.statsoft.com/products/statistica-12-newfeatures/

6. N.S. Altman, The American Statistician ,46 (3), 175-185 (1992)

7. V.Vapnik, Statistical Learning Theory (Wiley, New York, 1998)

8. L. Demidova, Yu. Sokolova, E. Nikulchev, International Review on Modelling and Simulations, 8 (4), 446-457 (2015)

9. L. Demidova, Yu. Sokolova, Development of the SVM Classifier Ensemble for the Classification Accuracy Increase, 6-th Seminar on Industrial Control Systems: Analisys, Modeling and Computing, ITM Web of Conference, 6, 02003 (2016).

10. L. Demidova, Yu. Sokolova, Training Set Forming For SVM Algorithm With Use Of The Fuzzy Clustering Algorithms Ensemble On Base Of Cluster Tags Vectors Similarity Matrices, 2015 International Conference "Stability and Control Processes" in Memory of V.I. Zubov (SCP), 619622 (2015)

11. L. Demidova, E. Nikulchev, Yu. Sokolova, International Journal of Advanced Computer Science and Applications, 7 (2), 16-24 (2016)

12. L. Demidova, Yu. Sokolova, Modification Of Particle Swarm Algorithm For The Problem Of The SVM Classifier Development, 2015 International Conference «Stability and Control Processes» in Memory of V.I. Zubov (SCP). pp. 623-627 (2015)

13. J.A. Hartigan, M.A. Wong, Journal of the Royal Statistical Society, Series C, 28(1), 100-108 (1979)

14. M.R. Gupta, Y. Chen, Theory and Use of the EM Algorithm (2010) 